\title{
LA ADQUISICIÓN O SUMINISTRO DE BIENES Y SERVICIOS DE CARACTERÍSTICAS TÉCNICAS UNIFORMES Y DE COMÚN UTILIZACIÓN A TRAVÉS DE SUBASTA INVERSA
}

\section{THE ACQUISITION OR SUPPLY OF GOODS AND SERVICES OF UNIFORM TECHNICAL CHARACTERISTICS AND OF COMMON USE THROUGH DUTCH AUCTIONS}

\author{
Jaime Eduardo Chaves Villada* \\ Fecha de recepción: 4 de agosto de 2009 \\ Fecha de aceptación: 12 de abril de 2010
}

* Abogado de la Pontificia Universidad Javeriana. Especialista en Derecho Administrativo y Derecho Sustantivo y Contencioso Constitucional de la Universidad Javeriana. Profesor de pregrado y posgrado en Derecho Constitucional y Contratación Estatal. Bogotá, Colombia.

Correo electrónico: jaimechavesv@gmail.com 


\section{RESUMEN}

El presente artículo busca demostrar el desbordamiento de la potestad reglamentaria del Gobierno nacional en el ámbito de la contratación estatal, específicamente a partir de las disposiciones establecidas en el Decreto 2474 del 2008 en lo que concierne a la adquisición y suministro de bienes y servicios de características técnicas uniformes y de común utilización bajo el procedimiento de subasta inversa, frente a los criterios de selección objetiva desarrollados en la Ley 1150 expedida en el año 2007.

Palabras clave: contratación estatal, criterios de selección objetiva, selección abreviada, subasta inversa, bienes y servicios de características técnicas uniformes y de común utilización.

\section{ABSTRACT}

The present article seeks to demonstrate the overflow of the regulation legal authority of the national government in the area of the state contracting, specifically from the regulations established in the Decree 2474 of 2008 regarding the acquisition and supply of goods and services of technical uniform characteristics and of common utilization under the procedure of inverse auction, opposite to the criteria of objective selection developed in the law 1150 sent in the year of 2007.

Key words: public contracting, objective selection criteria, abbreviate selection, Dutch auction, goods and services of uniform technical characteristics and of common use 


\section{INTRODUCCIÓN}

Desde la entrada en vigencia de la Ley 1150 expedida en el año $2007^{1}$, se han expedido veinte decretos reglamentarios, se han proferido ocho sentencias sobre el estudio de constitucionalidad de la ley y siete providencias del Consejo de Estado sobre el estudio de legalidad de las disposiciones reglamentarias, desatando una conmoción jurídica en el ámbito de la contratación estatal que sin lugar a dudas ha derivado en una gran preocupación para el cumplimiento de las funciones estatales.

Uno de los grandes cambios que ha generado tal conmoción, es la modificación de las modalidades de selección del contratista reguladas por el artículo $2^{\circ}$ de la Ley 1150 expedida en el 2007 y el cambio en la aplicación de los criterios de selección objetiva consagrados a partir del artículo $5^{\circ}$ de la misma ley. Situación que se constata en el hecho de que el Consejo de Estado se ha tenido que manifestar sobre la ilegalidad de disposiciones reglamentarias ya sea porque en algunos casos dicha reglamentación violaba las nuevas modalidades de selección de contratistas (licitación pública, contratación directa, concurso de méritos, selección abreviada) o bien porque con la misma se creaban modalidades de selección del contratista diferentes a las creadas por la ley.

Sin embargo, a pesar de la situación anterior, hasta este momento, no se ha estudiado por parte del máximo tribunal de lo contencioso administrativo, una inquietante figura que surge de la aplicación de los criterios de selección objetiva en las normas que reglamentan el procedimiento de evaluación en subasta inversa, implementado para la adquisición o suministro de bienes y servicios de características técnicas uniformes y de común utilización como causal de la selección abreviada ${ }^{2}$, de acuerdo con la cual la pluralidad de oferentes aparece como requisito fundamental para la realización de la subasta inversa. Una situación que sin duda obliga a cuestionarse sobre la validez del mismo, frente a los criterios de selección objetiva consagrados en el artículo $5^{\circ}$ de la Ley 1150 expedida en el 2001.

Lo anterior por cuanto de la situación se deriva una afectación directa del reglamento a la ley, puesto que la creación del requisito de pluralidad implica una búsqueda obligatoria de su cumplimiento ajena a las posibilidades de los oferentes, pero exigida con todo el rigor de la ley por parte de la entidad para avanzar en el procedimiento, que a su vez generan la creación de nuevas

1 Ley 1150 del 2007. Por medio de la cual se introducen medidas para la eficiencia y la transparencia en la Ley 80 de 1993 y se dictan otras disposiciones generales sobre la contratación con recursos públicos. Julio 16 del 2007. D.O. n 46.691.

2 Ley 1150 del 2007. Por medio de la cual se introducen medidas para la eficiencia y la transparencia en la Ley 80 de 1993 y se dictan otras disposiciones generales sobre la contratación con recursos públicos. Articulo $2^{\circ}$. numeral $2^{\circ}$. Julio 16 del 2007. D.O. n $^{\circ} 46.691$. 
etapas que incluso permitía la presentación de nuevas ofertas, lo cual excede desde todo punto de vista los parámetros establecidos por las leyes 80 de 1993 y 1150 del 2007.

Para demostrar esta posición, analizaremos en primer lugar los criterios de selección objetiva establecidos por la Ley 1150 del 2007 con su inmediato antecedente en la Ley 80 de 1993 y en segundo lugar la aplicación del procedimiento de una subasta inversa como mecanismo de adquisición o suministro de bienes y servicios de características técnicas uniformes y de común utilización, con el objeto de demostrar la afectación que sufren los criterios de selección objetiva por la creación del requisito de pluralidad que ha reglamentado el Gobierno nacional y que obligan apremiantemente a un pronunciamiento judicial que vele por el respeto de la legalidad del ordenamiento jurídico.

\section{LOS CRITERIOS DE SELECCIÓN OBJETIVA: "UNA NUEVA PERSPECTIVA"}

Es preciso esclarecer cuál es el papel preponderante que juegan los criterios de selección objetiva, puesto que son ellos los que utilizarán las entidades públicas para fundamentar los requisitos que soliciten a las ofertas que se fuesen a presentar en un determinado proceso de selección de contratistas. En este entendido, debemos esclarecer que el principio de selección objetiva consagrado bajo el régimen de la Ley 80 de 1993, es diferente a la regulación traída por la Ley 1150 del 2007, puesto que la primera (A) permitía más flexibilidad para determinar los criterios de selección que adoptarían las entidades públicas con respecto a la búsqueda de la oferta más favorable y, la segunda (B) regula de manera clara y taxativa cuáles son esos criterios de selección que se utilizarán para determinar la oferta más favorable. Razón por la cual en este momento las entidades no tienen la discreción de omitir o utilizar otros que no sean los contemplados por la ley y que de igual manera no son viables por la vía de la reglamentación crearlos, modificarlos o extinguirlos, puesto que no es competencia del Gobierno nacional ocuparse de dicha materia.

\section{La discrecionalidad contemplada en el artículo 29 de la Ley 80 expedida en $1993^{3}$}

El derogado artículo 29 de la Ley 80 expedida en 1993, establecía dos bases para el desarrollo de este principio: a) La obligación de selección objetiva, entendida como aquella evaluación que se realiza sobre una propuesta independientemente de intereses subjetivos de cualquier índole, y b) La oferta más

3 Ley 80 de 1993. Por medio de la cual se expide el Estatuto General de Contratación de la Administración Pública. Octubre 28 de 1993. D.O. n 41.094. 
favorable, donde el cumplimiento de los criterios de escogencia y factores de ponderación establecidos en los pliegos de condiciones, permitían establecer un orden de elegibilidad de las ofertas después de realizar el cotejo de las mismas por parte de los evaluadores designados por las entidades públicas en cada uno de los procesos de selección del contratista.

El Consejo de Estado bajo la vigencia del artículo 29 de la Ley 80 de 1993, en varias ocasiones se pronunció sobre el tema, pero vale la pena destacar el fallo del 3 de diciembre del 2007 como una de las últimas providencias en que el tribunal se refirió al respecto estudiando el Decreto 2170 del 2002 así:

"En este sentido, la selección objetiva consiste en la escogencia de la oferta más favorable para la entidad, siendo improcedente considerar para ello motivaciones subjetivas. Para tal efecto, con carácter enunciativo, la norma consagra factores determinantes para esa elección, los cuales deben constar de manera clara, detallada y concreta en el respectivo pliego de condiciones o términos de referencia, o en el análisis previo a la suscripción del contrato si se trata de contratación directa, y que sobre todo, deben apuntar al cumplimiento de los fines estatales perseguidos con la contratación pública. Lo anterior significa que en la contratación, sea mediante licitación o concurso públicos o contratación directa, la administración está obligada a respetar los principios que la rigen transparencia, economía y responsabilidad-y los criterios de selección objetiva establecidos en las bases del proceso para la escogencia del contratista al que se le adjudicará el contrato por haber presentado la mejor propuesta, tales como cumplimiento, experiencia, organización, equipos, plazo, precio, etc., los cuales deberán ser previamente analizados y evaluados por la entidad con arreglo a las condiciones del pliego que rigen el respectivo proceso, con el fin de determinar en forma motivada que la propuesta elegida resulta ser en realidad la más ventajosa, de suerte que el concepto jurídico indeterminado "oferta más ventajosa" del artículo 29 citado pueda ser susceptible de concreción en sede de revisión de la legalidad y razonabilidad de la comparación de los ofrecimientos, por cuanto esta actividad no es discrecional ni arbitraria, sino reglada, por cuanto pone a la administración en frente del inequívoco camino de elegir aquella oferta que se ajusta a dichas condiciones y adjudicar al proponente que supera a los demás. En consecuencia, la intención del legislador al definir legalmente este principio, fue la de regular la escogencia de la mejor oferta mediante una selección en la que prime la transparencia, imparcialidad e igualdad de oportunidades, y ajena a consideraciones subjetivas, para lo cual juegan un papel preponderante los factores y criterios de selección que en función a la específica necesidad haya fijado la administración en los respectivos pliegos de condiciones o términos de referencia".

Bajo esta normativa se daba pie para que las entidades tuvieran un margen de libertad en los criterios de selección adoptados para las evaluaciones, pues-

4 Consejo de Estado. Sala de lo Contencioso Administrativo. Sección Tercera. Proceso 24.715 (C.P. 
to que el fin sería siempre desarrollar una selección de la oferta más favorable pero los medios o criterios de selección serían determinados por la entidad según sus necesidades, lo cual, de plano, ya implicaba que la estructuración del proceso se fundamentara en los mejores criterios para cumplir con esta obligación, estableciéndose así una clara coherencia entre la ley y la realidad puesto que todos los procesos no son iguales y mucho menos los objetos contratados. Un criterio que, aunque en nuestro parecer era altamente favorable para el ejercicio de una administración más eficiente, fue abandonado con la reforma aportada por la Ley 1150 del 2007.

\section{La rigidez contemplada en el artículo $5^{\circ}$ de la Ley 1150 expedida en el $\mathbf{2 0 0 7}^{5}$}

La Ley 1150 expedida en el año 2007, en su artículo $32^{6}$ derogó completamente el artículo 29 de la Ley 80 expedida en 1993, determinando una nueva forma de aplicar la selección objetiva dentro de los procesos de selección de contratista, incorporando un mecanismo de evaluación sobre criterios de verificación (capacidad técnica, económica, jurídica y de organización) y de ponderación (factores técnicos y económicos) ${ }^{7}$, de tal forma que la entidad obligatoriamente debe cumplir con estos elementos sin tener la posibilidad de adicionar otros o de omitir alguno salvo en las excepciones previstas para concurso de méritos, puesto que incurriría en un incumplimiento de la ley y afectaría la selección objetiva que quiso establecer el legislador.

El Consejo de Estado, a propósito de la selección objetiva en la Ley 1150 del 2007, claramente describe la diferencia que emana del artículo $5^{\circ}$ entre criterios de verificación y criterios de ponderación como factores únicos y determinantes de la selección objetiva, así:

"Como fluye del tenor de esta disposición, que modificó el deber de selección objetiva, el Legislador distinguió entre requisitos habilitantes y factores de ponderación, previendo que los primeros versan sobre la capacidad jurídica y las condiciones de experiencia, capacidad financiera y de organización de los proponentes, los cuales serán objeto de verificación de cumplimiento (cumple

Ruth Stella Correa Palacio; diciembre 3 del 2007).

5 Ley 1150 del 2007. Por medio de la cual se introducen medidas para la eficiencia y la transparencia en la Ley 80 de 1993 y se dictan otras disposiciones generales sobre la contratación con recursos públicos. D.O. n ${ }^{\circ} 46.691$.

6 Ley 1150 del 2007. Por medio de la cual se introducen medidas para la eficiencia y la transparencia en la Ley 80 de 1993 y se dictan otras disposiciones generales sobre la contratación con recursos públicos. Artículo 32. D.O. no 46.691.

7 Ley 1150 del 2007. Por medio de la cual se introducen medidas para la eficiencia y la transparencia en la Ley 80 de 1993 y se dictan otras disposiciones generales sobre la contratación con recursos públicos. Artículo $5^{\circ}$. D.O. n ${ }^{\circ} 46.691$. 
o no cumple el oferente) para la participación en el proceso de selección y no otorgarán puntaje (pasa o no pasa esa primera etapa), con excepción de la experiencia que sí debe ser ponderada en tratándose de los procesos para selección de consultores mediante concurso de méritos.

Además, al proscribir las consideraciones o motivaciones subjetivas en el proceso de escogencia del contratista, la norma legal en comento consagró los criterios de selección propiamente dichos, esto es, los factores de calificación o ponderación a exigir en los pliegos de condiciones para determinar la oferta más favorable dependiendo del objeto que se va a contratar, así:

i) la oferta más favorable es aquella que resulta de la ponderación precisa y detallada de los factores técnicos y económicos contenidos en los pliegos de condiciones; pero, ii) en las contrataciones cuyo objeto sea la adquisición o suministro de bienes y servicios de características técnicas uniformes y común utilización, el único factor de "evaluación" (sic) será el menor precio ofrecido; y iii) en las contrataciones de consultores, esto es, en los concursos de mérito, los factores de calificación serán los destinados a valorar los aspectos técnicos de la oferta o proyecto, como también se podrán utilizar criterios de experiencia específica del oferente y del equipo de trabajo, pero en ningún caso se podrá incluir el precio como factor de escogencia". ${ }^{8}$

\section{Días después se volvió a recalcar por parte de la misma sala así:}

"La norma legal citada regula uno de los principios fundamentales de la contratación estatal: la selección objetiva, aspecto que por su importancia también fue tratado por la Ley 80 de $1993^{9}$, de manera que la nueva ley, por ocuparse del tema expresamente, derogó el artículo 29 de la anterior.

En efecto, la Ley 1150 introdujo cambios no sólo importantes y novedosos, sino que trazó un nuevo rumbo en esta materia, pues al paso que la Ley 80 de 1993 permitía a las entidades estatales definir, con amplísima libertad, como (sic) se podían evaluar las ofertas, la nueva norma impuso directrices bastante

8 Consejo de Estado. Sala de lo Contencioso Administrativo. Sección Tercera. Auto 36.476 (C.P. Ruth Stella Correa Palacio; abril $1^{\circ}$ del 2009).

9 El artículo 29 de la Ley 80 de 1993 disponía que: "La selección de contratistas será objetiva. Es objetiva la selección en la cual la escogencia se hace al ofrecimiento más favorable a la entidad y a los fines que ella busca, sin tener en consideración factores de afecto o de interés y, en general, cualquier clase de motivación subjetiva. Ofrecimiento más favorable es aquel que, teniendo en cuenta los factores de escogencia, tales como cumplimiento, experiencia, organización, equipos, plazo, precio y la ponderación precisa, detallada y concreta de los mismos, contenida en los pliegos de condiciones o términos de referencia o en el análisis previo a la suscripción del contrato, si se trata de contratación directa, resulta ser el más ventajoso para la entidad, sin que la favorabilidad la constituyan factores diferentes a los contenidos en dichos documentos, sólo alguno de ellos, el más bajo precio o el plazo ofrecido. El menor plazo que se ofrezca inferior al solicitado en los pliegos, no será objeto de evaluación. El administrador efectuará las comparaciones del caso mediante el cotejo de los diferentes ofrecimientos recibidos, la consulta de precios o condiciones del mercado y los estudios y deducciones de la entidad o de los organismos consultores o asesores designados para ello. En caso de comparación de propuestas nacionales y extranjeras, se incluirán los costos necesarios para la entrega del producto terminado en el lugar de su utilización." 
estereotipadas, que redundan en una disminución de la libertad, en manos de la administración, para establecer la forma como se pueden o deben evaluar las propuestas". ${ }^{0}$

Encontramos entonces que el artículo $5^{\circ}$ de la Ley 1150 expedida en el año $2007^{11}$ reguló el principio de selección objetiva, estableciendo para ello dos tipos de criterios de selección permanentes dentro de los procesos de contratación estatal, unos de verificación donde se evaluarán las ofertas presentadas conforme a la capacidad técnica, económica, jurídica y de organización y, otros de ponderación donde se calificarán factores técnicos y económicos, bajo los parámetros que en ambos casos se establezcan en los pliegos de condiciones definitivos de manera precisa y detallada para verificación de las ofertas y ponderación de las mismas.

Este cambio implica una limitación dentro del ámbito de discreción con que contaban las entidades públicas bajo el régimen de la Ley 80, donde la obligación preeminente era la búsqueda de la oferta más favorable exceptuando cualquier subjetivismo que desviase los fines estatales, dejando no obstante un campo de desempeño amplio para que la entidad determinara qué factores a su consideración, permitirían alcanzar dicho mandato, es decir, que para el legislador de 1993 bastó con imponer la obligación de buscar la oferta más favorable puesto que con toda razón ello ya implicaba de antemano crear criterios coherentes con el objeto de contratar bajo la luz de la selección objetiva.

A contrario sensu, la Ley 1150 del 2007 al fijar los factores que determinan la oferta más favorable limita el campo de discreción de los servidores públicos y los somete al imperio de la legalidad contemplado en el artículo $6^{\circ}$ de la Constitución Política de Colombia de 1991 y, por ende, impide que por vía de reglamentación se modifiquen, extingan o supriman dichos criterios.

\section{LA AFECTACIÓN DE LOS CRITERIOS DE SELECCIÓN OBJETIVA A PARTIR DEL PROCEDIMIENTO}

Los requisitos para la participación en un proceso de contratación estatal deben estar orientados al cumplimiento por parte del oferente interesado. Actualmente, como se explicó en el punto anterior, la ley establece los criterios para que a partir de allí se fundamenten los requerimientos necesarios que la entidad pueda solicitar, pero bajo unos imperativos establecidos a partir de los principios especiales que orientan la contratación estatal, como es el

10 Consejo de Estado. Sala de lo Contencioso Administrativo. Sección Tercera. Auto 36.054 (C.P. EnRiQue Gil Botero; mayo 27 del 2009).

11 Ley 1150 del 2007. Por medio de la cual se introducen medidas para la eficiencia y la transparencia en la Ley 80 de 1993 y se dictan otras disposiciones generales sobre la contratación con recursos públicos. Artículo $5^{\circ}$. D.O. n ${ }^{\circ} 46.691$. 
caso del principio de transparencia consagrado en el artículo 24 de la Ley 80 de $1993^{12}$, bajo el cual se exige que dichos requisitos entre otras cosas no sean "condiciones y exigencias de imposible cumplimiento, ni exenciones de la responsabilidad derivada de los datos, informes y documentos que se suministren"13, pues bien, en el procedimiento de subasta inversa bajo estudio se solicita una pluralidad de oferentes, sin la cual no se podrá realizar la subasta inversa, de tal modo que si un oferente queda habilitado por presentar su oferta en los términos y condiciones que la entidad haya solicitado pero no hay otra oferta habilitada, causa dos consecuencias inmediatas: a) la entidad no podrá evaluar su oferta en los factores de ponderación; b) la pluralidad se convierta en un requisito adicional a su oferta para poder acceder a la etapa de adjudicación del proceso. Situaciones que claramente afectan los criterios de selección objetiva puesto que este requisito no tiene sustento alguno en aquéllos y es de imposible cumplimiento para el oferente.

\section{El desbordamiento de la potestad reglamentaria en el marco general del procedimiento}

El artículo 22 del Decreto 2474 del $2008,{ }^{14}$ reglamentó el procedimiento de evaluación en la subasta inversa como mecanismo de adquisición o suministro de bienes y servicios de características técnicas uniformes y de común utilización, en los siguientes términos:

"Artículo 22. Verificación de los requisitos habilitantes. Para que una subasta pueda llevarse a cabo en los términos de este decreto deberán resultar habilitados para presentar lances de precios por lo menos dos (2) proponentes.

El resultado de la verificación de los requisitos habilitantes se publicará de conformidad con lo señalado en el artículo $8^{\circ}$ del presente decreto. En dicho informe se señalarán los proponentes que no se consideran habilitados y a los cuales se les concederá un plazo para que subsanen la ausencia de requisitos o la falta de documentos habilitantes, so pena del rechazo definitivo de sus propuestas. Luego de verificados y subsanados los requisitos habilitantes, si a ello hubiere lugar, las entidades procederán a llevar a cabo la subasta dentro de los plazos fijados en los pliegos de condiciones.

Si sólo un oferente resultare habilitado para participar en la subasta, la entidad ampliará el plazo para la presentación de los documentos habilitantes y la oferta

12 Ley 80 de 1993. Por medio de la cual se expide el Estatuto General de Contratación de la Administración Pública. Octubre 28 de 1993. D.O. n 41.094.

13 Ley 80 de 1993. Por medio de la cual se expide el Estatuto General de Contratación de la Administración Pública. Artículo 24, numeral 5ㅇ, literal d). Octubre 28 de 1993. D.O. nº 41.094.

14 Decreto 2474 del 2008 [Departamento Nacional de Planeación]. Por medio del cual se reglamentan parcialmente la Ley 80 de 1993 y la Ley 1150 del 2007 sobre las modalidades de selección, publicidad, selección objetiva y se dictan otras disposiciones. Artículo 22. Julio 7 del 2008. 
inicial de precio, por el término indicado en los pliegos de condiciones, el cual en ningún caso podrá ser mayor de la mitad del inicialmente previsto.

Si vencido ese plazo no se alcanza la pluralidad de proponentes, la entidad adjudicará el contrato al proponente habilitado, siempre que su oferta no exceda el presupuesto oficial indicado en el pliego de condiciones.

Parágrafo. Durante la prórroga a que hace referencia el tercer inciso del presente artículo, cualquier interesado 15 podrá presentar oferta, incluyendo aquellos proponentes que fueron considerados no hábiles para participar en la subasta, en los términos señalados en el presente artículo".16

En el primer inciso de la disposición se establece que por lo menos dos oferentes deberán estar habilitados para la realización de una subasta inversa, lo que con seguridad parecería obvio y apenas lógico de entender, puesto que no podría realizarse una puja dinámica de reducción sucesiva de precios en los términos del artículo 18 del Decreto 2474 del $2008^{17}$ con un solo oferente habilitado, respondiendo al elemento público y concurrencial necesario para su realización.

No obstante lo anterior, como ya hemos recalcado, la selección objetiva adoptada por el artículo $5^{\circ}$ de la Ley 1150 del $2007^{18}$ establece los criterios de selección objetiva que deben cumplir los oferentes para orientar la escogencia del ofrecimiento más favorable, consagrando unos criterios de verificación como requisitos habilitantes y unos criterios de ponderación de factores técnicos y económicos, en virtud de los cuales la entidad podrá calificar la mejor propuesta y en esa medida establecer el orden de elegibilidad entre quienes hayan sido habilitados y hayan obtenido la mejor puntuación, pero en el numeral tercero hace referencia específica a la adquisición o suministro de bienes y servicios de características técnicas uniformes y común utilización, determinando que el único factor de evaluación será el menor precio ofrecido, con justa razón, puesto que no será posible ponderar factores técnicos porque estos son de características uniformes.

Ahora bien, la pluralidad de oferentes solicitada por el primer inciso del artículo 22 del Decreto 2474 del 2008 para la realización de una subasta inversa, crea un elemento nuevo para la búsqueda de la oferta más favorable, es decir, la pluralidad de oferentes es un requisito consagrado en el proceso

15 Aparte declarado ilegal mediante Sentencia del Consejo de Estado. Sala de lo Contencioso Administrativo. Sección Tercera. Expediente 36.054 (C.P. EnRIQUe Gil Botero; abril 14 del 2010).

16 Decreto 2474 de 2008 [Departamento Nacional de Planeación]. Por medio del cual se reglamentan parcialmente la Ley 80 de 1993 y la Ley 1150 de 2007 sobre las modalidades de selección, publicidad, selección objetiva y se dictan otras disposiciones. Artículo 22. Julio 7 del 2008.

17 Decreto 2474 del 2008 [Departamento Nacional de Planeación]. Por medio del cual se reglamentan parcialmente la Ley 80 de 1993 y la Ley 1150 del 2007 sobre las modalidades de selección, publicidad, selección objetiva y se dictan otras disposiciones. Artículo 18. Julio 7 del 2008.

18 Ley 1150 del 2007. Por medio de la cual se introducen medidas para la eficiencia y la transparencia en la Ley 80 de 1993 y se dictan otras disposiciones generales sobre la contratación con recursos públicos. Artículo $5^{\circ}$. Julio 16 del 2007. D.O. nº 46.691. 
de subasta inversa pero que se convierte en un factor determinante para la selección del contratista en la medida que de no cumplirse se deberá extender el proceso de selección, obligando a que en el caso donde se presente una única propuesta habilitada, el oferente debe esperar el agotamiento de otros términos en búsqueda de competidores para poder llegar a su ponderación económica.

El problema de fondo recae en un desbordamiento de la potestad reglamentaria del Gobierno nacional, ya que exigir dentro de este procedimiento una pluralidad de oferentes, implica contrariar por parte de la entidad los criterios de selección objetiva vigentes para la contratación estatal, ya que ese requisito no encuentra sustento en ellos, es más, el sistema de evaluación tal como lo ha entendido el Consejo de Estado en el marco de estudio del artículo 12 numeral $3^{\circ}$ del Decreto 2474 del 2008 es materia de ley, no susceptible de reglamentación gubernamental, por lo que se cierra la posibilidad tanto para las entidades públicas como también para el Gobierno nacional crear, modificar o suprimir lo ya establecido por la ley. Resaltemos lo que determinó el Consejo de Estado:

"De ese modo, el reglamento olvidó que el deber de selección objetiva es una materia desarrollada por la Ley 1150 del 2007 en el artículo $5^{\circ}$ y, por lo mismo, cuando el artículo 12, numeral $3^{\circ}$, letra b), del Decreto 2474 del 2008, a través del cual se reglamentó "el deber de selección objetiva", estableció un sistema de evaluación diferente a los previstos por la ley, modificó los términos y alcances de la norma legal, razón por la cual incurrió el Gobierno nacional en un desbordamiento de su facultad reglamentaria.

En conclusión, la disposición demandada resulta contraria a la Constitución Política, pues no puede el Gobierno nacional establecer modificaciones a la forma de evaluación de las propuestas en ejercicio de la potestad reglamentaria, toda vez que el artículo 273 superior reservó al legislador la definición de "la manera como se efectuará la evaluación de las propuestas", razón por la cual resulta procedente acceder a la medida cautelar solicitada". ${ }^{19}$

Y de la misma manera, sobre potestad reglamentaria del Gobierno en materia de contratación estatal, se ha sostenido lo siguiente:

"Por eso, ha subrayado el Consejo de Estado que al Gobierno nacional en ejercicio de esta potestad en el ámbito de la contratación pública no le es permitido desbordar los mandamientos del legislador y extender sus efectos a supuestos no previstos en la norma legal reglamentada; o expedir una reglamentación que no

19 Consejo de Estado. Sala de lo Contencioso Administrativo. Sección Tercera. Auto 36.601 (C.P. Mauricio Fajardo; mayo 27 del 2009). 
haga más expedita o facilite la aplicación de la ley; o tipificar negocios jurídicos civiles, comerciales o administrativos, cuyos elementos esenciales y naturales corresponde a la ley y nunca al reglamento; o fijar restricciones que cercenen las opciones legales, como aquella según la cual las partes pueden acordar el modo de costear la publicación del contrato; o conferir facultades de índole judicial, como cuando en el artículo 17 del Decreto 856 de 1994 se sugirió que las cámaras de comercio tenían competencia para determinar los perjuicios y costas causados y relacionados con la impugnación en materia de registro de proponentes, entre otros eventos" 20 .

\section{El desbordamiento de la potestad reglamentaria en la aplicación concreta del procedimiento}

El inciso tercero del artículo 22 del Decreto 2474 del 2008, establece que si sólo un oferente resultare habilitado, se deberá ampliar el plazo para la presentación de los documentos habilitantes y la oferta inicial de precio, para que las personas que se hayan presentado puedan subsanar su oferta y participar en el proceso, en este momento adquiere relevancia el parágrafo del mismo artículo, por virtud del cual en este mismo plazo se daba la opción para que "cualquier" interesado pudiera presentar ofertas, lo cual fue estudiado por el máximo tribunal estableciendo lo siguiente:

No obstante lo anterior, el principio de libertad de concurrencia debe a su vez ponderarse con el principio de igualdad, ello implica que la multiplicidad de oferentes que se busca participen en el proceso de selección deben caracterizarse por tener un punto común de partida; es decir que, aunque lo ideal es la participación de todos los individuos que puedan presentar una oferta a la Administración, este ideal puede llevarse a cabo solamente si la inclusión de los mismos no implica una diferencia de trato que sea injustificada. En otros términos: tratándose de la ampliación del plazo para la presentación de aquellos documentos que soportan los denominados criterios habilitantes, resulta contrario al principio de igualdad abrir la puerta del procedimiento de selección indiscriminadamente a personas que (sic) un principio no manifestaron su interés de participar en el mismo. En este evento, el punto de partida no es equiparable a la situación de aquellos que en el momento oportuno hicieron explícito su deseo de participar e intentaron habilitarse. ${ }^{21}$

No obstante lo anterior, el problema sigue vigente. Veamos un ejemplo:

a) Se establece como plazo de cierre del proceso el día 15 de marzo del 2010;

b) Se presentan A y B;

20 Consejo de Estado. Sala de lo Contencioso Administrativo. Sección Tercera. Proceso 24.715 (c.P. Ruth Stella Palacio Correa; diciembre 3 del 2007).

21 Sentencia del Consejo de Estado. Sala de lo Contencioso Administrativo. Sección Tercera. Expediente 36.054 (C.P. Enrique Gil Botero; abril 14 del 2010). 
c) En el informe de evaluación la entidad determina que la propuesta de A pasó la evaluación sobre los factores de verificación y la propuesta de $\mathrm{B}$ deberá ser subsanada en el término de cinco días;

d) Al cabo de ese término, B no subsana;

e) Por virtud del inciso primero del artículo 22 bajo estudio, la entidad no puede realizar la subasta inversa;

f) La entidad deberá ampliar el plazo en los términos establecidos por el inciso tercero en mención;

g) El oferente A, que ha cumplido con los requisitos establecidos por la entidad en los pliegos de condiciones tiene la obligación de mantener su oferta en esta amplitud de plazo, deberá esperar los resultados de la evaluación y al final sabrá si es posible la realización de la subasta inversa o si la entidad ya puede aplicar la disposición del inciso cuarto para verificar la idoneidad de su oferta económica y de ser el caso adjudicar el contrato.

Se evidencia fácilmente que dicho procedimiento viola flagrantemente el principio de economía desarrollado por el artículo $3^{\circ}$ del Código Contencioso Administrativo, ${ }^{22}$ puesto que no se cumple con la obligación de adelantar los procedimientos en el menor tiempo posible o con la menor cantidad de gastos de quien interviene en ellos, se atenta contra el principio de economía consagrado en el numeral primero del artículo 25 de la Ley 80 de $1993^{23}$, por el cual se establece que dentro de un proceso de contratación estatal los términos son preclusivos y las etapas perentorias dejando sin competencia a la entidad para crear o revivir etapas y términos ya agotados, como es la etapa de presentación de las ofertas ${ }^{24} \mathrm{y}$ sin lugar a dudas la disposición crea una desigualdad en la ley al imponerle una carga adicional al oferente cumplido, sin observar fines razonables y constitucionales, como lo ha exigido la Corte Constitucional de Colombia ${ }^{25}$.

22 Código Contencioso Administrativo [CCA]. Decreto 01 de 1984. Artículo $3^{\circ}$. Enero 10 de 1984. D.O. $n^{\circ}$. 36.439. (Colombia).

23 Ley 80 de 1993. Por la cual se expide el Estatuto General de la Contratación de la Administración Pública. Artículo 25. Octubre 28 de 1993. D.O. nº 41.094.

24 Véase al respecto Luis Guillermo Dávila Vinueza. Régimen jurídico de la contratación estatal. Pág. 189. Ed., Legis. 2a . Edición. (2003).

25 Véase al respecto: Corte Constitucional de Colombia Sentencia C-540 del 2008 (м.P. Humberto Antonio Sierra Porto: 28 de mayo del 2008). 


\section{CONCLUSIón}

La nueva normativa en materia de contratación estatal, es una completa conmoción jurídica que necesita urgentemente ser atendida. El Consejo de Estado ha hecho lo posible por preservar los principios que orientan la contratación pública en cada uno de los temas que se ha puesto a su consideración, por lo que el llamado de atención debe estar directamente enfocado a las autoridades que tienen la competencia para desarrollar las disposiciones reglamentarias, siendo necesario realizar una completa evaluación de las novedades introducidas por la Ley 1150 expedida en el 2007 para asimilar las consecuencias que ello trae y así en un marco de coherencia reglamentarlos.

Los organismos de control jugarán un papel muy importante puesto que deberán ser consecuentes en sus investigaciones de responsabilidad disciplinaria y fiscal frente a las actuaciones de los funcionarios públicos, ya que al encontrarnos en tan difícil etapa de construcción normativa las interpretaciones jurídicas salen a relucir, pero el principio de buena fe y de transparencia serán los determinantes para sus decisiones en tanto en cuanto no se creen criterios unificadores de aplicación normativa.

Seguramente muchos serán los campos determinantes en el buen desarrollo de la contratación estatal de Colombia, pero lo que sí aparece claro, es la necesidad de construir entre todos una sólida plataforma normativa que permita el cumplimiento de las finalidades estatales y de una búsqueda de crecimiento económico que permita mejorar la calidad de los servicios públicos a cargo del Estado, puesto que de no realizarse dicho consenso la eficacia, transparencia, economía, igualdad y demás principios rectores de la contratación estatal quedarán relegados a un segundo plano y fuera del contexto que a partir de la Constitución Política de Colombia de 1991 se estableció. 


\section{BIBLIOGRAFÍA}

\section{Constitución}

Constitución Política de Colombia de 1991. Artículo $6^{\circ}$.

\section{Leyes}

Ley 153 de 1887. Que adiciona y reforma los códigos nacionales.

Ley 61 de 1886 y 57 de 1887, artículo $2^{\circ}$. Agosto 24 de 1887. D.O. n ${ }^{\circ} 7.151$ y 7.152.

Ley 80 de 1993. Por la cual se expide el Estatuto General de la Contratación de la Administración Pública, artículo 25. Octubre 28 de 1993. D.O. n 41.094.

Ley 1150 del 2007. Por medio de la cual se introducen medidas para la eficiencia y la transparencia en la Ley 80 de 1993 y se dictan otras disposiciones generales sobre la contratación con recursos públicos. Julio 16 del 2007. D.O. nº 46.691.

\section{Códigos}

Código Contencioso Administrativo [CCA]. Decreto 01 de 1984, artículo $3^{\circ}$. Enero 10 de 1984. D.O. $\mathrm{n}^{\circ}$ 36.439. (Colombia).

\section{Decretos}

Decreto 2170 del 2002 [Departamento Nacional de Planeación]. Por el cual se reglamenta la Ley 80 de 1993, se modifica el Decreto 855 de 1994 y se dictan otras disposiciones en aplicación de la Ley 527 de 1999. Septiembre 30 del 2002.

Decreto 66 del 2008 [Departamento Nacional de Planeación]. Por medio del cual se reglamenta la Ley 1150 del 2007 sobre las modalidades de selección, publicidad y selección objetiva y se dictan otras disposiciones. Enero 16 del 2008.

Decreto 2474 del 2008 [Departamento Nacional de Planeación]. Por medio del cual se reglamentan parcialmente la Ley 80 de 1993 y la Ley 1150 del 2007 sobre las modalidades de selección, publicidad, selección objetiva y se dictan otras disposiciones. Julio 7 del 2008.

Decreto 2025 del 2009 [Departamento Nacional de Planeación]. Por el cual se modifica parcialmente el Decreto 2474 del 2008 y se dictan otras disposiciones. Junio 3 del 2009.

Decreto 3576 del 2009 [Departamento Nacional de Planeación]. Por el cual se modifica parcialmente el Decreto 2474 del 2008 y el Decreto 2025 del 2009.

\section{Doctrina}

Luis Guillermo Dávila Vinueza. Régimen jurídico de la contratación estatal. Pág. 189. $2^{\mathrm{a}}$ Edición. Ed. Legis. (2003).

\section{Jurisprudencia}

Consejo de Estado Sala de lo Contencioso Administrativo. Sección Tercera

Proceso 24.715 (C.P. Ruth Stella Correa Palacio; diciembre 3 del 2007).

Auto 36.476 (C.P. Ruth Stella Correa Palacio; abril $1^{\circ}$ del 2009).

Auto 36.054 (C.P. Enrique Gil Botero; mayo 27 del 2009).

Auto 36.601 (C.P. Mauricio FAJARdo; mayo 27 del 2009). 
Sentencia 36.054 (C.P. EnRiQue Gil Botero; abril 14 del 2010).

Corte Constitucional de Colombia

Sentencia C-540 del 2008 (m.p. Humberto Antonio Sierra Porto: mayo 28 del 2008). 